\title{
Dark Matter Searches with GLAST
}

\author{
E.Nuss * \\ LPTA Montpellier II University \\ on behalf of GLAST LAT Dark Matter and New Physics WG
}

\begin{abstract}
The Gamma-Ray Large Area Space Telescope (GLAST), scheduled to be launched in fall 2007, is the next generation satellite for high-energy gamma-ray astronomy. The Large Area Telescope (LAT), GLAST main instrument, with a wide field of view ( $>2 \mathrm{sr})$, a large effective area $\left(>8000 \mathrm{~cm}^{2}\right.$ at $\left.1 \mathrm{GeV}\right)$ and $20 \mathrm{MeV}-300$ $\mathrm{GeV}$ energy range, will provide excellent high energy gamma-ray observations for Dark Matter searches. In this paper we examine the potential of the LAT to detect gamma-rays coming from WIMPS annihilation in the context of supersymmetry. As an example, two search regions are investigated: the galactic center and the galactic satellites.
\end{abstract}

Key words: Dark Matter, New Physics, Gamma-rays

\section{Introduction}

The Cold Dark Matter (CDM) problem is one of the most fascinating and intriguing issue in present day cosmology. It has been a subject of special interest to high-energy physicists, astrophysicists and cosmologists for many years. According to a wealth of observations and arguments, such as excessive peculiar velocities of galaxies within clusters of galaxies or gravitational arcs, it can make up a significant fraction of the mass of the universe. On the galactic scale, dark matter halos are required to explain the observed rotation curves in spiral galaxies or the velocity dispersion in elliptical galaxies. Virtually all proposed candidates require physics beyond the standard model of particle physics and could be detected through stable products of

\footnotetext{
* Corresponding author

Email address: eric.nuss@lpta.in2p3.fr (E.Nuss ).
} 
Table 1

Summary of the main LAT Science Performances relevant for Dark Matter searches

\begin{tabular}{|ll|}
\hline Parameter & Current Best Estimate \\
\hline Peak Effective Area (in range 1-10 GeV) & $\simeq 9000 \mathrm{~cm}^{2}$ \\
Energy Resolution $10 \mathrm{GeV}$ on-axis & $<6 \%$ \\
PSF $68 \% 10 \mathrm{GeV}$ on-axis & $<0.1^{0}$ \\
Field of View & $>2 \mathrm{Sr}$ \\
Dead Time & $26.5 \mu \mathrm{sec} /$ event nominal \\
\hline
\end{tabular}

their annihilations: energetic neutrinos, antiprotons, positrons, gamma-rays etc. Supersymmetric extensions of the standard model of particle physics provide a natural candidate for CDM in the form of a stable uncharged Majorana fermion (neutralino).

Hereafter, we briefly report on the potential of the GLAST high-energy gammaray telescope to detect Dark Matter indirectly through their annihilation in the halo of the galaxy and in clumpy substructures throughout the Galactic halo.

\section{The Gamma-ray Large Area Space Telescope (GLAST) mission}

GLAST is the next-generation gamma-ray telescope for studying high-energy gamma-ray emission from astrophysical sources 1 . Its main instrument, the Large Area Telescope (LAT), is a modular 4x4-tower pair-conversion telescope instrumented with a plastic anticoincidence shield which vetoes charged cosmic rays, a tracker of silicon strip planes with foils of tungsten converter followed by a segmented CsI electromagnetic calorimeter. A photon traversing the tracker will have high probability of converting into the tungsten foils, thus forming an electron-positron pair, subsequently tracked by the silicon strip detectors. The reconstructed trajectories of this pair, together with their energy deposition in the calorimeter, allows to reconstruct the direction and energy of the incident gamma-ray photon. The main characteristics of the LAT detector, i.e. the effective area, point spread function and energy dispersion, have been obtained from detailed Monte Carlo studies and parameterized by a series of functions: the Instrument Response Function (IRF) 2. The main LAT Science Performances relevant for Dark Matter searches are summarized in Table 1. The LAT takes much of its basic design concept from its predecessor EGRET but the energy range (20 MeV-300 GeV and above), field-of-view

1 For more details, see the GLAST website at: http://glast.gsfc.nasa.gov/

2 http://www-glast.slac.stanford.edu/software/IS/glast_lat_performance.htm 


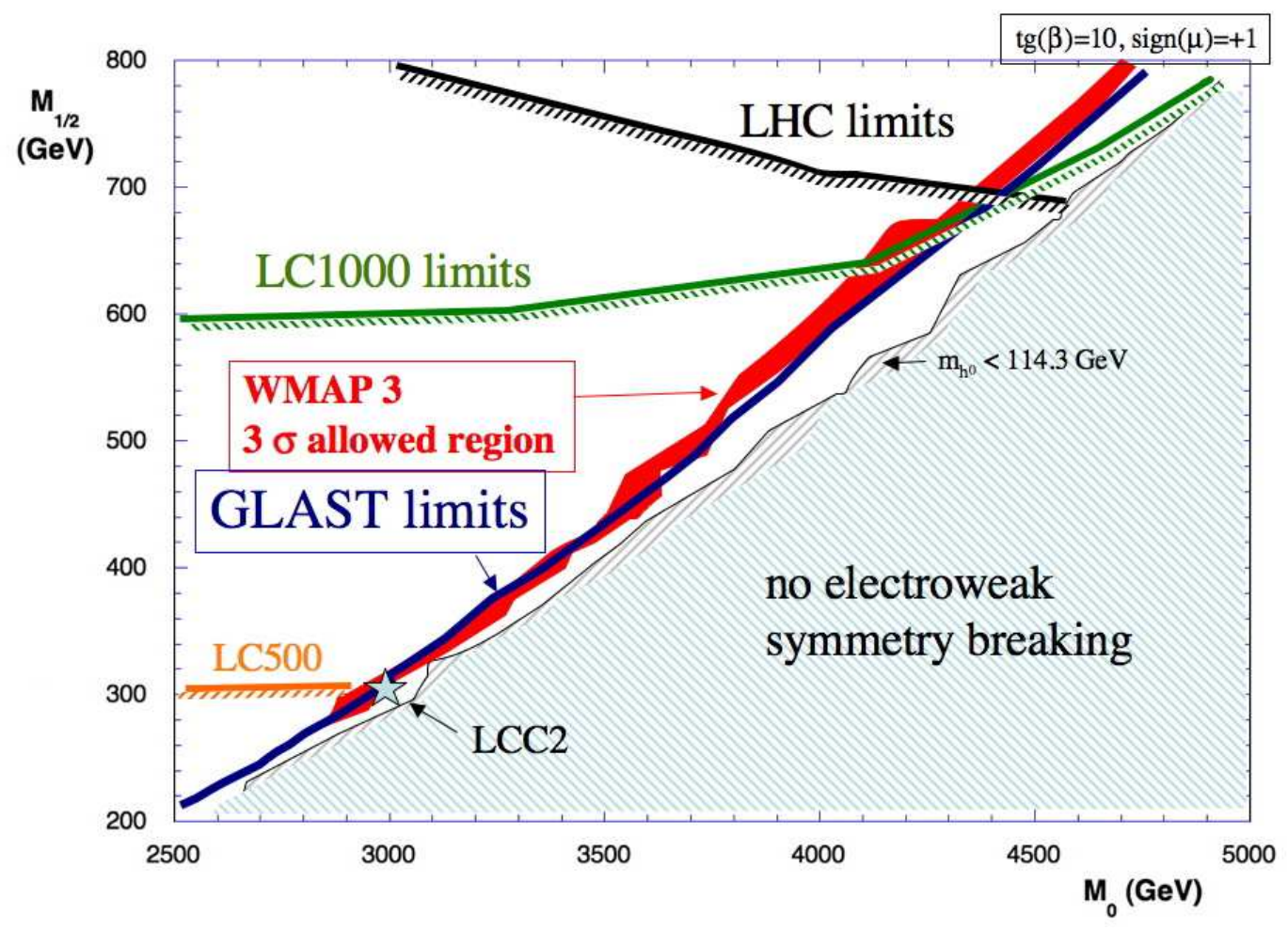

Fig. 1. GLAST $5 \sigma$ sensitivity to Dark Matter signal for 5 years observation of the Glactic Center in the mSUGRA $\left(m_{0}, m_{\frac{1}{2}}\right)$ plane for $\tan (\beta)=10, A_{0}=0, \mu>0$ and top quark mass $m_{t}=174 \mathrm{GeV}$.

(greater than 2 steradians) and angular resolution will provide the LAT with a factor $\sim 30$ better sensitivity. This improvement should allow to detect several thousands of new high-energy sources and shed light on many issues left open by EGRET.

A detailed description of GLAST science prospects and an introduction to the experiment can be found in Michelson (2001). The LAT is now completed and handed to the spacecraft vendor for integration. It represents the largest silicon strip detector ever built for space applications.

GLAST is scheduled for launch in fall 2007.

\section{Dark Matter searches with GLAST at the Galactic Center}

The unusual point source found by EGRET at or near the Galactic Center gives a strong indication of an excess with respect of the standard model production of gamma-rays. The spectrum of this potential source of WIMP annihilation is extremely hard, non-power law, and the source is possibly ex- 


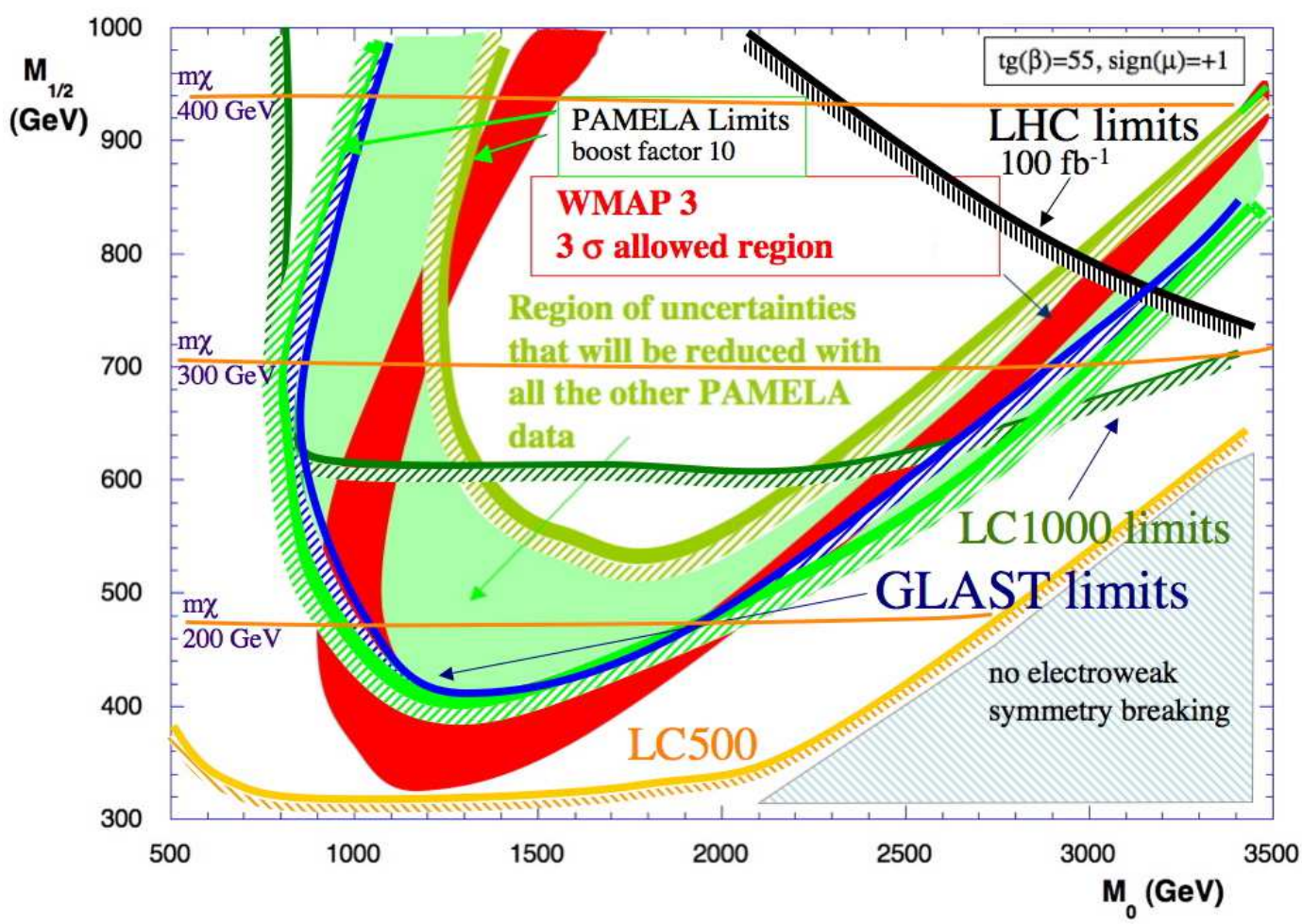

Fig. 2. GLAST $5 \sigma$ sensitivity to Dark Matter signal for 5 years observation of the Glactic Center in the mSUGRA $\left(m_{0}, m_{\frac{1}{2}}\right)$ plane for $\tan (\beta)=55, A_{0}=0, \mu>0$ and top quark mass $m_{t}=174 \mathrm{GeV}$.

tended (Mayer-Hasselwander, et.al. 1998). Several hypotheses for the origin of this source have been proposed and the dark matter hypothesis is not yet ruled out.

The figures (1) and (2) shows the mSUGRA $\left(m_{0}, m_{\frac{1}{2}}\right)$ plane with parameters similar to the two mSUGRA benchmark points LCC2 2 and LCC4 respectively, as defined in Baltz et al. (2006). The GLAST $5 \sigma$ sensitivity to dark matter signal (continuum spectra) for 5 years observation of a $0.1^{0}$ region around the galactic center is shown at the blue lines and below. The diffuse background was estimated using the GALPROP code v50 3 (point source substracted). The GLAST sensitivity is compared with mSUGRA accelerator limits from Baer et al. (2004). The dark matter halo used to compute the GLAST indirect search sensitivity is a truncated Navarro Frank and White (NFW) halo profile (as defined in Baltz et al. (2006)). The methodology for deriving the various curves is presented in Cesarini et al. (2004). The PAMELA antiprotons limits are from Lionetto et al. (2005). In these figures, it can be seen that GLAST can explore a good portion of the supersymmetric parameter space for this kind of halo.

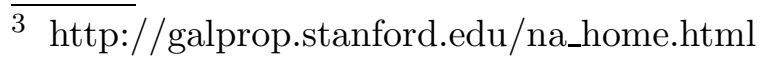




\section{Dark Matter searches with GLAST in Galactic Satellites}

According to N-body simulations on test particles with only gravitational interactions (see Navarro et al. (1997), Moore et al. (1999)), WIMPs are expected to form high density dark matter substructures in the galactic halo with masses less than approximately $10^{7-8}$ solar masses.

The number of Milky Way dark matter satellites observable by GLAST have been estimated using a semianalytic method Tavlor and Babul (2004) where the dark matter satellite distribution is roughly spherically symmetric about the galactic center and extends well beyond the solar orbit; thus the dark matter satellites are located mostly at high galactic latitudes.

The figure (3) shows the number of dark matter clumps observable by GLAST for 5 years observation for two benchmark mSUGRA points as a function of the significance. The black line corresponds to simulations for the LCC4 mSUGRA model defined in Baltz et al. (2006) and the grey line corresponds to the LCC2 model. The LCC1 and LCC3 models are much less favorable, with no clumps detected. The background was estimated using the EGRET point source subtracted sky map above $1 \mathrm{GeV}$.

\section{Conclusions}

In this paper we presented a preliminary GLAST-LAT sensitivity to indirect gamma-ray signature of WIMP annihilation, based on the best simulations currently available to the LAT collaboration. As an example, two search regions have been investigated, the Galactic Center and the galactic satellites. Despite its dependence on a still unprecisely known background, our estimate shows that the GLAST telescope, should be capable of searching for Dark Matter in the energy range $E_{\gamma} \geq 20 \mathrm{MeV}$. The peculiar timing, spectral, and spatial signatures associated to WIMP annihilations will permit to reject other possible astrophysical sources of gamma-rays.

\section{References}

H. Baer, A. Belyaev, T. Krupovnickas et al. , JCAP 0408 (2004) 005

E. A. Baltz, M. Battaglia, M. E. Peskin et al, hep-ph/0602187

A.Cesarini, F.Fucito, A.Lionetto et al, Astroparticle Physics 21, 267-285, 2004.

A.Lionetto, A.Morselli, V.Zdravkovic et al., Astropart. Phys. JCAP09(2005)010. 


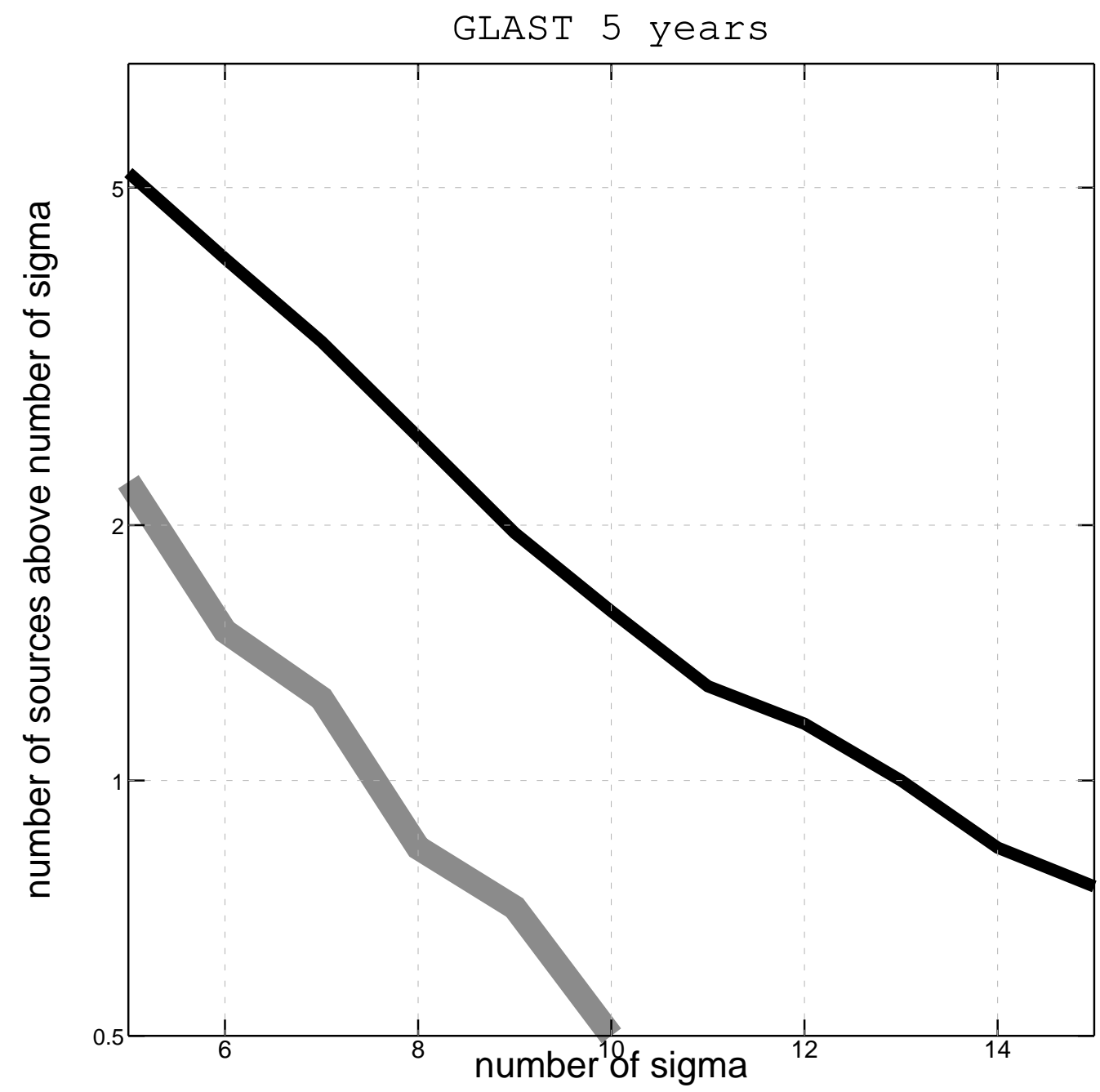

Fig. 3. Number of clumps observed by GLAST vs. number of sigma significance in 5 years of all-sky scanning.

H. A. Mayer-Hasselwander et al., Astron. \& Astrophys. 335, 161 (1998).

P.F. Michelson, "The Gamma-ray Large Area Space Telescope Mission: Science Opportunities" in AIP Conf. Proc. 587: Gamma 2001: Gamma-Ray Astrophysics, 2001, pp.713-+.

B. Moore, T. Quinn, F. Governato, J. Stadel and G. Lake, Mon. Not. R. Astron. Soc. 310, 1147 (1999).

J. F. Navarro, C. S. Frenk and S. D. M. White, Astrophys. J. 490, 493 (1997).

J. E. Taylor and A. Babul, Mon.Not.Roy.Astron.Soc.364, 2005 and references therein 